\title{
EFFECT OF CHITOSAN PRETREATMENT OF THE DEMINERALIZED DENTIN SURFACE ON THE DURABILITY OF RESIN DENTIN BONDING. AN IN VITRO STUDY
}

\author{
Mostafa Saber Mohamed Ata*
}

\begin{abstract}
Abstract: the aim of this study was to evaluate the effect of pretreatment of the demineralized dentin surface with Chlorohexidine (CHX), Chitosan (Ch), riboflavin (RF) and Chitosan/riboflavin (RF) combination on the shear bond strength and short-term bond durability with total etch adhesives. Fifty sound extracted human permanent molars were used in this study. The teeth were embedded in plastic tube containers and a flat dentin surface was then prepared by grinding the occlusal enamel exposing superficial dentin surfaces. Specimens were etched for $15 \mathrm{sec}$ with $35 \%$ phosphoric acid and were then randomly divided into five groups (10 teeth each), one control group and four experimental groups according to the surface pretreatment (Chlorohexidine, Chitosan, riboflavin and Chitosan/riboflavin combination). This is followed by application of total etch adhesive system and resin composite restoration. The resin composite samples were then divided to be stored either for $24 \mathrm{~h}$ or 6 months in distilled water at $37 \mathrm{C}$ followed by shear bond strength testing using Instron Testing Machine. The data were analyzed using ANOVA test. The results revealed that, pretreatment of the demineralized dentin surface with $\mathrm{CHX}, \mathrm{Ch}, \mathrm{RF}$, and $\mathrm{Ch} / \mathrm{RF}$ 1:1 significantly improved the shear bond strength mean values at both $24 \mathrm{~h}$ and 6 months storage in distilled water compared to the control group. Within control group, significant reduction in shear bond strength mean values were found after 6 months storage in distilled water compared to the baseline measurements at $24 \mathrm{~h}$. The immediate pretreatment of the demineralized dentin with collagen cross linkers could be safely recommended as an effective chairside procedure to improve shear bond strength of resin composite to dentin.
\end{abstract}

KEYWORDS: Chitosan, Dentin bonding, resin composite

\section{INTRODUCTION}

Achieving a stable and efficient bond between composite resin and dentin still remains a challenge in restorative dentistry. Some of the recently reported factors that occur during bonding to dentin and could result in acceleration of the degradation of the collagen phase and consequently reducing the durability of the bonded interface are insufficient resin impregnation of dentin collagen, high

\footnotetext{
* Associate Professor of Operative Dentistry, Faculty of Dental Medicine, Al-Azhar University
} 
permeability of the bonded interface and activation of endogenous collagenolytic enzymes ${ }^{(\mathbf{1})}$.

To improve the durability of resin/dentin interface, several studies have attempted to reinforce and stabilize the collagen fibrils through inter-and intra-molecular crosslinking, aiming to decrease the enzymatic degradation and subsequently a more efficient demineralized dentin collagen substrate ${ }^{(2)}$.

Pretreatment of the dentin surface with agents that inhibit the activity of MMPs could improve bond durability. Chlorohexidine strongly inhibits the proteolytic activities of MMP-2, 8 , and $9^{(3)}$.

Chitosan is a natural biocompatible polymer, has a great antimicrobial activity, able to form a micro and nano-fibrillar network of high mechanical properties, and has been investigated as a reinforcement phase of collagen network in tissue engineering research ${ }^{(4)}$.

Recently, riboflavin showed to be an effective crosslinking agent by its ability to produce free radicals when photoactivated with spectral range from ultra violet to visible light ${ }^{(5)}$.

Therefore, the aim of this study was to evaluate the effect of pretreatment of the demineralized dentin surface using Chlorohexidine, Chitosan, riboflavin and Chitosan/riboflavin combination on the shear bond strength and short-term bond durability with total etch adhesives.

\section{MATERIALS AND METHODS}

Preparation of the chitosan/riboflavin formulations: all solutions were prepared in the Faculty of Pharmacy, Biochemistry Department, AL-Azhar University.

Solution of Chitosan (Ch): Solution of Chitosan powder $(1 \%)$ was prepared by dissolving one gram of chitosan in form of powder in $100 \mathrm{Ml}$ of distilled water. Chitosan was purified by dissolving in 1 $\mathrm{mol} / \mathrm{L}$ acetic acid, filtered and precipitated three times in $1 \mathrm{~mol} / \mathrm{L} \mathrm{NaOH}$. The precipitate was washed with ethanol/water mixtures $(70 / 30 \mathrm{v} / \mathrm{v})$, freezedried and then solutions of Chitosan (1\%) were prepared in acetic acid $(1 \mathrm{~mol} / \mathrm{L})$.

Solution of riboflavin: Solution of riboflavin powder $(1 \%)$ was prepared by dissolving one gram of riboflavin in form of powder in $100 \mathrm{Ml}$ of distilled water.

Ch/RF combination: was prepared by adding the Chitosan solution to the riboflavin solution at 1:1 (with Ch content of $50 \mathrm{v} / \mathrm{v} \%$ ). The $\mathrm{pH}$ of all solutions was maintained at approximately 6 to avoid aggregation of chitosan particles. All solutions were kept in light-proof test tubes to avoid any light activation of riboflavin before use.

\section{Specimen preparation}

Fifty sound extracted human permanent molars were used in this study. The extracted teeth were stored in $0.1 \%$ thymol solution immediately after extraction for 2 weeks then in distilled water at room temperature for not more than one month. ${ }^{(6)}$

The teeth were embedded in plastic tube containers (30 mm length and $10 \mathrm{~mm}$ diameter) containing cold cure acrylic resin so that the occlusal surfaces projected above the resin through the upper end while the lower end of the tube is resting on a glass slab surface. Immediately after embedding, a flat dentin surface was then prepared by grinding the occlusal enamel using a low-speed diamond saw (Buehler, Lake Bluff, IL, USA) under water cooling exposing superficial dentin surfaces approximately $1 \mathrm{~mm}$ below DEJ. Exposed dentin surface was then wet-polished with increasing grit-sizes from 600 $1000 \mathrm{SiC}$ papers to create a standardized smear layer (Carbimet; Buehler, USA). Specimens were etched for $15 \mathrm{sec}$ with $35 \%$ phosphoric acid and thoroughly rinsed with distilled water for $10 \mathrm{sec}$. The excess water was removed with absorbent paper leaving hydrated dentin surface. The teeth were randomly divided into five groups (10 teeth each), one control group and four experimental groups. 
Group 1 (n=10): control: No pretreatment was done on the etched dentin surface. Only dentin bonding agent and composite resin were applied.

Group 2 (n=10): $2 \%$ CHX solution pretreatment; $10 \mathrm{~mm}$ of $2 \%$ Chlorohexidine digluconate solution (Neel Kink Healthcare, Put.Safe PUS, India) was applied to the etched dentin surface for $30 \mathrm{sec}$ and then dried with absorbent paper.

Group 3 (n=10): Ch pretreatment; $10 \mathrm{~mm}$ of $1 \%$ Chitosan solution was applied to the etched dentin surface for $30 \mathrm{sec}$ and then dried with absorbent paper.

Group 4 (n=10): RF pretreatment; $10 \mathrm{~mm}$ of $1 \%$ riboflavin solution was applied to the etched dentin surface for $30 \mathrm{sec}$ and then photoactivated by conventional blue light curing unit of $600 \mathrm{~mW} / \mathrm{cm} 2$ output for $20 \mathrm{sec}$.

Group 5 (n=10): $\mathbf{C h} / \mathbf{R F}$ pretreatment; $10 \mathrm{~mm}$ of $1 \%$ Chitosan/riboflavin solution (1:1) was applied to the etched dentin surface for $30 \mathrm{sec}$ and then photoactivated by conventional blue light curing unit of $600 \mathrm{~mW} / \mathrm{cm} 2$ output for $20 \mathrm{sec}$.

After pretreatment of the demineralized dentin surfaces of the specimens, two consecutive coats of dentin bonding agent (AdperTM Singlebond; 3 M ESPE, USA) were applied to the moist dentin surfaces for $15 \mathrm{sec}$, gently air dried for $5 \mathrm{sec}$ and light cured for $10 \mathrm{sec}$ using dental blue light curingunit (Curing Light 2500; 3 M ESPE, MN, USA) with an output of $600 \mathrm{~mW} / \mathrm{cm} 2$ and at a distance of $10 \mathrm{~mm}$ from the light source.

After bonding, a standardized Teflon plate $(8 \mathrm{~mm}$ diameter and $5 \mathrm{~mm}$ height) containing two holes (2 $\mathrm{mm}$ diameter with $2 \mathrm{~mm}$ intervening between the two holes) were fixed on the flat occlusal surface of each specimen to build-up the composite resin. The composite resin (Filtek Z350 XT A3; 3 M ESPE) was then built-up in 3 equal increments into the fixed Teflon plate holes and photo-cured with the same blue light curing unit for $20 \mathrm{sec}$ for each increment. After removal of the Teflon plate, two composite studs ( $2 \mathrm{~mm}$ diameter and $5 \mathrm{~mm}$ height for each stud) on each flat occlusal dentin surface were obtained. Another photo-curing of each stud was done with the same blue light curing unit for $20 \mathrm{sec}$.

Specimens were stored for $24 \mathrm{~h}$ in distilled water at $37 \mathrm{C}$ to complete the polymerization reaction before shear bond strength testing. The obtained resin composite studs were then divided to be stored either for $24 \mathrm{~h}$ or 6 months in distilled water at 37 C $(n=10)$. The distilled water was changed every week for the 6 months storage period. One stud of each tooth was tested for shear bond strength after 24 hours and the other stud after 6 months. An Instron Universal Testing Machine (Zwick Universal Testing Machine, Material prufung, 2675, Germany) containing knife edged rod at a crosshead speed of $0.5 \mathrm{~mm} /$ minute was used to test the debonding strength. All data tests were analyzed using one-way ANOVA followed by Turkey's-multiplecomparison post hoc test for pair-wise comparison between the different groups. P-values less than 0.05 are considered significant.

\section{RESULTS}

The results of the statistical analysis of the shear bond strength mean values for both $24 \mathrm{~h}$ and 6 months storage in distilled water are shown in Table 1. Pretreatment of the demineralized dentin surface with $\mathrm{CHX}, \mathrm{Ch}, \mathrm{RF}$, and $\mathrm{Ch} / \mathrm{RF}$ 1:1 significantly improved the shear bond strength mean values at both $24 \mathrm{~h}$ and 6 months storage in distilled water compared to the control group. However, no statistical significant differences in shear bond strength were found among all pretreated dentin surface groups after 6 months of storage periods. Within control group, significant reduction in shear bond strength mean values were found after 6 months storage in distilled water compared to the baseline measurements at $24 \mathrm{~h}$. 
TABLE (1)

\begin{tabular}{|c|c|c|}
\hline Groups & $24 \mathrm{H}$ & 6 months \\
\hline Control (C) & $22.9 \pm 5.654^{\mathrm{a}}$ & $15.2 \pm 4.297^{\mathrm{b}}$ \\
\hline Chlorohexidine (ChX) & $27.8 \pm 4.821^{\mathrm{c}}$ & $27.1 \pm 5.216^{\mathrm{c}}$ \\
\hline Chitosan (Ch) & $28.7 \pm 4.917^{\mathrm{c}}$ & $27.2 \pm 4.163^{c}$ \\
\hline Riboflavin ( Rf) & $38.4 \pm 5.391^{\mathrm{d}}$ & $38.1 \pm 3.719^{d}$ \\
\hline $\begin{array}{c}\text { Chitosan/ Riboflavin 1:1 } \\
\text { (Ch/Rf) }\end{array}$ & $37.6 \pm 5.632^{\mathrm{d}}$ & $36.2 \pm 3.372^{\mathrm{d}}$ \\
\hline
\end{tabular}

Mean \pm standard deviations (SD) of shear bond strength in MP of different study groups stored in distilled water for 24 hours and 6 months at $37^{\circ} \mathrm{C}$

Groups with the same letters are statistically non significant (Tukey's test $p \leq 0.05$ )

Diagram showing shear bond strength in MP of different study groups stored in distilled water for 24 hours and 6 months at $37^{\circ} \mathrm{C}$

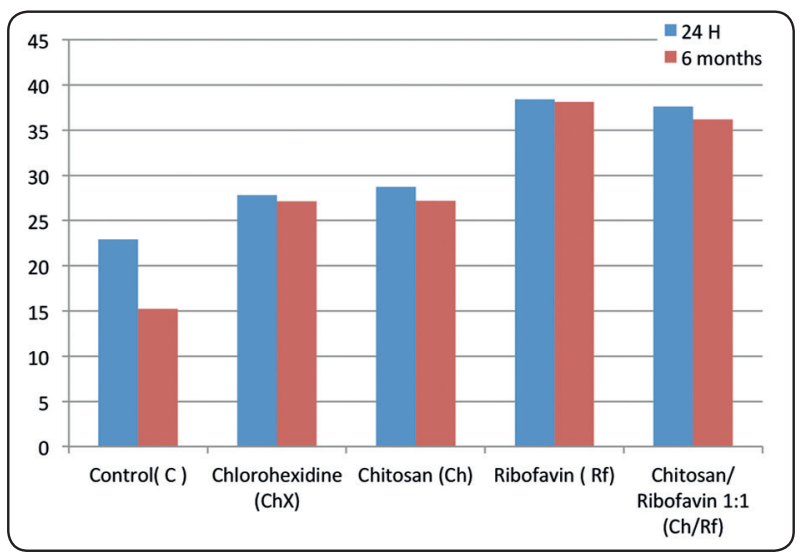

\section{DISCUSSION}

It was reported that dentinal fluid can act as a peroxide and oxygen reservoir, which may be present at the bonding interface, inhibit the polymerization reaction and reduce bond strength. Thus, application of antioxidant as a dentin pretreatment may be indicated to neutralize the free oxygen present in a higher amount in dentin than in enamel. ${ }^{(7)}$
Inter and intra-molecular dentin collagen crosslinking and reinforcement could increase bond strength and durability of resin dentin interface with time against enzymatic and / or hydrolytic degradation. Crosslinking agents as Glutaraldehyde might inactivate MMPs of dentin by crosslinking of their peptide chains, after acid demineralization, causing loss of molecular mobility but its cytotoxicity is a matter of concern ${ }^{(8)}$.

The results of this study revealed that, all groups showed significantly increased bond strength to dentin compared with control group.

Significant decrease in the shear bond strength found in control group after 6 month storage compared to the baseline measurement at $24 \mathrm{~h}$ (Table 1) may be related to the degradation of both of collagen and/or resin phases at resin/ dentin interface which increases the water content at the bonded interface leading to collagen degradation and subsequent detrimental effect on the longevity of resin/dentin bond ${ }^{(9)}$.

The increase in bond strength of specimens treated with $2 \%$ Chlorohexidine (CHX) may be related to its MMP inhibitory properties which prevent the binding of metal ions such as zinc or calcium, to MMPase, this inhibits its catalytic activity. More recently, 2\% Chlorohexidine (CHX) also inhibits dentinal Cysteine, Cathepsins B, K and L. When matrix metalloproteinase's (MMPs; 2,3,8,9,20 and Cathepsins) contained in dentin are activated by acid etching, the collagen fibrils are degraded slowly ${ }^{(\mathbf{1 0}, \mathbf{1 1})}$.

Chitosan was employed in the construction and modification of collagen scaffolds with improvement in the mechanical properties and degradation resistance. This is due to its biocompatibility and the ability to form fibrillar arrangements embedded in the protein matrix such as collagen. ${ }^{(12)}$

In an acidic media, chitosan provides more amino groups which enhance collagen crosslinking with riboflavin. These amino groups of chitosan capture the hydrogen ions producing positive charges result- 
ing in bioadhesive capability against the negatively charged substrates such as collagen ${ }^{(13)}$

Significant decrease in the shear bond strength found in specimens treated with chitosan group may be related to aggregation and retention of chitosan within the structure of the collagen fibrils network that result in obliteration of the interfibrillar spaces. It is well known that for successful bonding to dentin, open interfibrillar spaces and proper resin infiltration of the demineralized intertubular dentin collagen fibrils network to form a hybrid layer are required. ${ }^{(14)}$

The $1 \%$ solution only of riboflavin was used in this study, as higher concentrations of riboflavin will result in yellowish discoloration that might adversely affect any subsequent esthetic restoration and also affect the polymerization of the adhesive monomers. ${ }^{(15)}$

The results of this study showed that pretreatment of demineralized dentin surface with $1 \%$ riboflavin considerably increases the shear bond strength compared to the control group (Table 1). This enhancement in bond strength could be due to the crosslinking effect of riboflavin and the inhibition of MMPs activity. This is in agreement with Srinivasulu and others ${ }^{\left({ }^{(16)}\right.}$. They revealed that, when riboflavin is photoactivated with UVA or visible light, it generates free-radicals Like $\mathrm{O}^{2}$ and $\mathrm{O}^{-2}$. These free radicals form covalent cross links between adjacent collagen molecules and inhibition of MMPs activity.

This crosslinking effect of collagen fibrils by photo-activated riboflavin and inhibition of MMPs activity might also be one of the reasons for the higher shear bond strength stability found in this study, especially after 6 months storage in distilled water in comparison to the control group. Conventional blue light curing units versus UV might be possible alternative due to its ready availability and its safe use in dentistry. ${ }^{(17-18)}$

This study showed that pretreatment of demineralized dentin surface with chitosan/riboflavin combination increased the shear bond strength mean values. Reinforcement by adding a mechanically stronger component to collagen matrix as chitosan is another possible approach to improve the physico-mechanical properties of collagen scaffold. ${ }^{(19)}$

The significant improvement in shear bond strength found with $1: 1 \mathrm{Ch} / \mathrm{RF}$ surface pretreatment could be partially attributed to the reinforcement effect of chitosan in addition to riboflavin effect as mentioned before. ${ }^{(20)}$ Therefore, the positive dual effect of chitosan and riboflavin together on bonding to dentin could be revealed. In addition, when chitosan was added to riboflavin at 1:1 volume ratio, it did not obliterate interfibrillar spaces and adversely affect resin infiltration and hybrid layer formation.

Hence, the results of this study suggested that optimum results for the increased dentin bond strength can be achieved through out the immediate demineralized dentin pretreatment with collagen cross linkers could be safely recommended as an effective chairside procedure to improve shear bond strength of resin composite to dentin.

\section{CONCLUSION}

1. Within the limitation of this in vitro study, significant improvement in the bond strength of resin composite to dentin using total etch adhesive system was obtained when the dentin was pretreated with $2 \%$ Chlorohexidine, $1 \%$ riboflavin, chitosan, and 1:1 chitosan /riboflavin compared with control group.

2. Pretreatment of demineralized dentin surfaces with chitosan/riboflavin is not only of potential in adhesive dentistry, but could also be extended to other dental applications owing to the proven biological benefits of chitosan.

3. Chitosan/riboflavin combination might be able to overcome shortfalls in the current perspectives for improving bond durability through understanding factors affecting the long-term bonding performance of modern adhesives. 
4. Further studies regarding the effect of other variables such as different chitosan formulations, different adhesive systems and more prolonged and clinically relevant storage time, media and conditions, should be considered in the future.

\section{REFERENCES}

1. Frassetto A, Breschi L, Turco G, Marchesi G, Tay F, et al. (2016). Mechanism of degradation of the hybrid layer in adhesive dentistry and therapeutic agents to improve bond durability. Dental Materials. 32: 41-53

2. Bedran-Russo A, Pereira P, Duarte W, Drummond J, Yamauchi M. (2007). Application of cross linkers to dentin collagen enhances the ultimate tensile strength. Journal of Biomedical Materials Research Part B, Applied Biomaterials. 80: 268-272.

3. Deng D, Huan X, Huang C, Yang T, Du X, Wang Y, et al. (2013). Effects of Chlorohexidine on bonding durability of different adhesive systems using a novel thermocycling method. Aust Dent. 58: 148-55.

4. Dash M, Chiellinia F, Ottenbrite R. (2011). Chitosan-A versatile semi-synthetic polymer in biomedical applications. Progress in Polymer Science. 36: 981-1014.

5. Cova A, Breschi L, Nato, F, Ruggeri J, Carrilho M, Tja derhane L. et al. (2011). Effect of UVA-activated riboflavin on dentin bonding. Journal of Dental Research. 90: 14391445 .

6. Yang B, Adelungb R, Ludwiga K, Bobmann K. Pashley D, Kern M. (2005). Effect of structural change of collagen fibrils on the durability of dentin bonding. Biomaterials D J. 26: 5021-5031.

7. Breschi L, Mazzoni A, Ruggeri A, Cadenaro M, Lenarda R, Stefano E. (2008). Dental adhesion review: aging and stability of the bonded interface. Dental Materials: Official Publication of the Academy of Dental Materials. 24: 90-101.

8. Bedran-Russo A, Pashley D, Agee K, Drummond J, Miescke K. (2008). Changes in stiffness of demineralized dentin following application of collagen cross linkers. Journal of Biomedical Materials Research Part B, Applied Biomaterials. 86: 330-334.

9. Pashley D, Tay F, Breschi L, Tjaderhane L, Carvalho R, Carrilho M, Tezvergil, M. (2011). State of art of etch-and- rinse adhesives. Dental Materials: Official Publication of the Academy of Dental Materials. 27: 1-16.

10. Zhang S, and Kern M. (2009). The role of host-derived dentinal matrix metalloproteinase's in reducing dentin bonding of resin adhesives. International Journal of Oral Science. 1: 163-176.

11. Scaffa PM, Vidal CM, Barros N, Gesteria TF (2012). Chlorohexidine inhibits the activity of dental cysteine cathepsins. J Dent Res. 91: 420-5

12. Aryaei A, Jayatissav C, Jayasuriya A. (2012). Nano and micro mechanical properties of uncross-linked and crosslinked chitosan films. Journal of the Mechanical Behavior of Biomed Dent. Materials. 5: 82-89.

13. Stamford Arnaud, de Barros Neto B, Diniz J. (2010). Chitosan effect on dental enamel de-remineralization: an in vitro evaluation. Journal of Dentistry. 38: 848-852.

14. Petri D, Donegá J, Benassi AM, Bocangel H. (2013). Preliminary study on chitosan modified glass ionomer restoratives. Dental Materials. 23: 1004-1010.

15. Markowitz K and Rosenblum M.A. (2010). The effect of cationic polymer treatment on dye staining and on the adhesion of charged particles to dentin. Archives of Oral Biology. 55: 60-67.

16. Srinivasulu, Sidhya S, sujatha M, Mahalaximi S. (2013). Effect of collagen cross linkers on shear bond strength of a self-etch adhesive system to deep dentin. J Conserv Dent. 16:135-8

17. Daood U, Iqbal K, Nitisusanta L, Fawzy, AS. (2013). Effect of chitosan / riboflavin modification on resin / dentin interface: Spectroscopic and microscopic investigations. J Biomed Mater Res. 101: 1846-56

18. Poptani B, Gohil KS, Ganijwale J, Shkla M. (2012). Microtensile dentin bond strength of fifth with five seventhgeneration dentin bonding agents after thermocycling: an in vitro study. Contem Clin Dent 3 : 167-71

19. Shrestha A, Friedman S, Kishen A. (2011). Photodynamically crosslinked and chitosan- incorporated dentin collagen. J Dent Res. 90 : 1346-51

20. Madhavan K, Belchenko D, Motta, A, Tan W. (2010). Evaluation of composition and crosslinking effects on collagen-based composite constructs. Acta Biomaterial. 6: 1413-1422. 